\title{
Leptomycin, an antifungal and antitumor Streptomyces metabolite, is a novel chemical probe for eukaryotic nucleo-cytoplasmic transport
}

\author{
Minoru Yoshida*, Nobuaki Kudo, Hiroshi Taoka, Shin Akakura, \\ and Sueharu Horinouchi
}

\author{
Department of Biotechnology, Graduate School of Agriculture and Life Sciences, \\ The University of Tokyo, Bunkyo-ku Tokyo 113, Japan
}

(Received Octo. 14, 1998)

\begin{abstract}
Leptomycin B (LMB) is a Streptomyces metabolite that causes specific inhibition of the cell cycle of yeast and mammalian cells. The target molecule of LMB in fission yeast has been shown by genetic analysis to be CRM1, a highly conserved protein in eukaryotes. We isolated a human homolog of CRM1 (hCRM1) which was expressed ubiquitously in human shown to be a red that hCRM1 was essential for nuclear export of proteins. CRM1 was shown to be a receptor for the nuclear export signal (NES) of proteins in both yeast and mammalian cells. LMB binds directly to CRM1, which resulted in dissociation of the NES from the nuclear export machinery containing CRM1. Thus, LMB serves as a potent tool for understanding the molecular mechanisms of nucleo-cytoplasmic transport of proteins.
\end{abstract}

\section{Introduction}

Streptomyces produces a variety of metabolites, some of which are employed as chemical probes for analysis of coordinated mechanism to control cellular responses in higher eukaryotes as well as drugs for therapeutics. Eukaryotic cells receive a variety of positive and negative signals from external (growth factors, stresses, etc.) and internal (DNA damages, microtubule integrity, etc.) conditions. These signals are eventually transduced to the nucleus by importing the regulatory protein(s) into the nucleus. Accumulating evidence indicates the importance of nucleocytoplasmic transport of proteins across the nuclear envelope in regulating signal transduction and cell cycle progression. Specific signal sequences found in proteins such as a basic amino acid-rich nuclear localization signal (NLS) and a leucine-rich nuclear export signal (NES) are required for the energy-dependent nucleocytoplasmic transport. Recently, leptomycin B (LMB), which had been discovered as a potent antifungal and antitumor antibiotic, was shown to be a specific inhibitor of nuclear export of proteins. LMB facilitates dissection of the mechanism by which NES-bearing proteins are exported from the nucleus. In this review, we focus on the identification of CRM1 as an export receptor, the mechanism of inhibition of nuclear export of proteins by LMB, and regulated subcellular localization of several important proteins, which was highlighted by LMB.

\section{Discovery of leptomycins and related compounds \\ Leptomycins A and B (Fig. 1) produced by Streptomyces sp. ATS 1287 were originally dis-}

\footnotetext{
*Correspondence: Minoru Yoshida

Department of Biotechnology, Graduate School of Agriculture and Life Sciences, The University of Tokyo, Yayoi 1-1-1, Bunkyo-ku, Tokyo 113-0032, Japan. Tel: +81-3-3812-2111 ext. 5124, Fax: +81-3-3812-0544, E-mail: ayoshida@hongo.
ecc.u-tokyo.ac.jp
} 
covered by Beppu et al. ${ }^{1)}$ during the course of screening for antifungal antibiotics. The chemical structures of these compounds are unsaturated long-chain fatty acids with a terminal $\delta$ lactone ring ${ }^{2)}$. Leptomycin B (LMB) was the major active compound that inhibited the fungal growth. A characteristic feature of these compounds was the ability to induce morphological abnormalities in yeast and fungi such as cell elongation of the fission yeast Schizosaccharomyces pombe ${ }^{3)}$. $\mathrm{LMB}$ also causes in vitro $\mathrm{G} 1$ cell cycle arrest in various mammalian cells at nanomolar concentrations and in vivo antitumor activity against several murine experimental tumors $^{4.5)}$. Following the discovery of leptomycins, several derivatives such as kazusamy-cins and anguinomycins were reported (Fig. 1), some of which showed potent antitumor activity ${ }^{6,7)}$.<smiles>[R]C=C(C)CC(C)C(O)C(C)C(=O)C([R8])C=C(C)C=CCC(C)C=C([R])C=CC1OC(=O)C=CC1[R]</smiles>

\begin{tabular}{lllll} 
Leptomycin A & $\mathrm{R}_{1}$ & $\mathrm{R}_{2}$ & \multicolumn{1}{c}{$\mathrm{R}_{3}$} & \multicolumn{1}{c}{$\mathrm{R}_{4}$} \\
Leptomycin B & $\mathrm{CH}_{3}$ & $\mathrm{CH}_{3}$ & $\mathrm{CH}_{3}$ & $\mathrm{COOH}$ \\
Kazusamycin A & $\mathrm{CH}_{3}$ & $\mathrm{CH}_{2} \mathrm{CH}_{3}$ & $\mathrm{CH}_{3}$ & $\mathrm{COOH}$ \\
Kazusamycin B & $\mathrm{CH}_{3}$ & $\mathrm{CH}_{2} \mathrm{CH}_{3}$ & $\mathrm{CH}_{2} \mathrm{OH}$ & $\mathrm{COOH}$ \\
Anguinomycin A & $\mathrm{CH}$ & $\mathrm{CH}_{3}$ & $\mathrm{CH}_{2} \mathrm{OH}$ & $\mathrm{COOH}$ \\
Anguinomycin B & $\mathrm{H}$ & $\mathrm{CH}_{3}$ & $\mathrm{CH}_{3}$ & $\mathrm{COOH}$ \\
Anguinomycin C & $\mathrm{H}$ & $\mathrm{CH}_{2} \mathrm{CH}_{3}$ & $\mathrm{CH}_{3}$ & $\mathrm{COOH}$ \\
Anguinomycin D & $\mathrm{H}$ & $\mathrm{CH}_{3}$ & $\mathrm{CH}_{3}$ & $\mathrm{CH}_{3}$ \\
Leptolstatin & $\mathrm{H}$ & $\mathrm{CH}_{3}$ & $\mathrm{CH}_{3}$ & $\mathrm{CH}_{3}$ \\
Reductoleptomycin A & $\mathrm{CH}_{3}$ & $\mathrm{CH}_{3}$ & $\mathrm{CH}_{3}$ & $\mathrm{CH}_{2} \mathrm{OH}$ \\
& & & $\mathrm{CH}_{2} \mathrm{OH}$
\end{tabular}

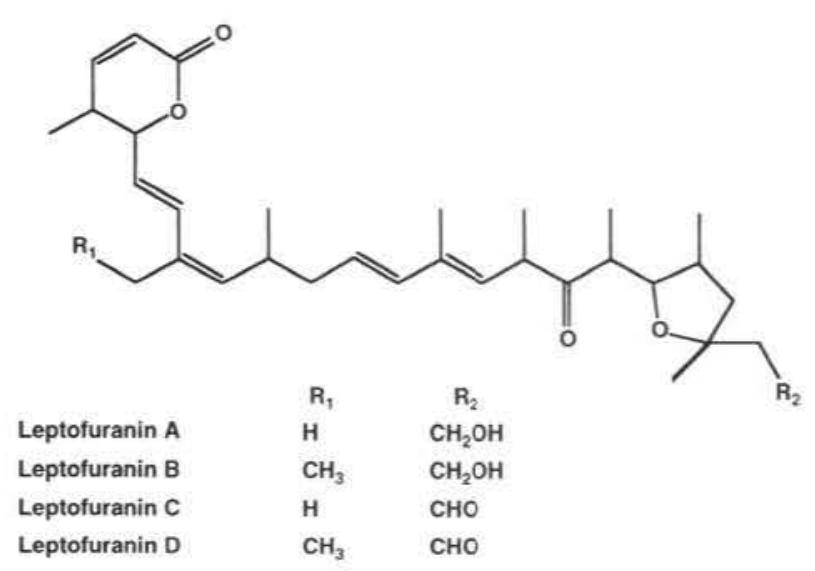

Fig. 1. Structures of leptomycins and their related compounds. 


\section{Identification of CRM1 as the target molecule of leptomycins}

Potent antiproliferative activity of LMB against both lower and higher eukaryotes suggests that the target molecule of LMB is highly conserved in eukaryotes and plays an important role in cell cycle control. To approach to the target, we took advantage of the fission yeast molecular genetic system, since $S$. pombe is one of the most sensitive organism to LMB. We isolated two LMB-resistance genes from an LMB-resistant mutant of $S$. pombe, which conferred LMB resistance on wild-type $S$. pombe. One gene $\left(p m \mathrm{ml}^{+}\right)$was found to be a novel homolog of mammalian MDR1, which pumps a variety of drugs out of cells in an energydependent manner ${ }^{8}$. overexpression of $\mathrm{pmdl}^{+}$conferred resistance to several structur-ally unrelated antifungal drugs. The other gene was a mutant of $\mathrm{crml}^{+}$, which had been originally reported as a gene essential for chromosome region maintenance. Coldsensi-tive $\mathrm{cml}$ mutants were isolated during visual screening by means of DAPI staining, which showed deformed filamentous or fragmented nuclear structures at the restrictive temperature ${ }^{9}$. The $\mathrm{crml}^{+}$gene encodes a 115 $\mathrm{kDa}$ protein that is essential for proliferation of $S$. pombe and is localized in the nucleus and its periphery. $\mathrm{Crm} 1$ is involved in the regulation of not only nuclear structure but also specific gene activity; the $\mathrm{crml}$ mutants produced an increased amount of p25 whose expression is regulated by an AP-1-like transcription factor. Genetic analysis demonstrated that $\mathrm{Crml}$ was a negative regulator for Pap1, the $S$. pombe AP-1 homolog ${ }^{10,11)}$. The wild-type $\mathrm{crml}^{+}$gene could also give resistance to LMB when introduced into the cells of the wild-type background with a multicopy vector. In contrast, the coldsensitive mutant crml-809 showed hypersensitivity to $\mathrm{LMB}$ at the permissive temperature. Furthermore, treat-ment of wildtype $S$. pombe with LMB caused abnormal nuclear morphology and p25 overproduction, which were almost identical to the terminal phenotypes of $\mathrm{crml}$ mutants. Taken together, we have proposed that $\mathrm{Crm} 1$ or its regulatory cascade is the cellular target of LMB. However, the molecular function of $\mathrm{Crm} 1$ was still unclear.

We next cloned cDNA encoding a functional mammalian homolog of $\mathrm{Crm1}$. The cloned cDNA, named human CRM1 (hCRM1), was able to complement the $S$. pombe $\mathrm{crm} /$ mutation and to cause growth inhibition and morphological abnormalities in $S$. pombe when overproduced. The mammalian CRMI gene was ubiquitously transcribed in all the tissues tested and its transcription level in cultured cells was regulated during the cell cycle. hCRM1 expressed in mammalian cells localized preferentially in the nuclear envelope. Recently, Wolff et $a l^{14)}$ reported that LMB inhibited nuclear export of the human immunodeficiency virus type 1 (HIV-1) Rev protein and Revdependent mRNA. Rev contains an NES which may be recognized by the host nuclear export machinery ${ }^{15}$. It seems possible that mammalian CRM1 is involved in nuclear export of proteins and that the cell cycle arrest of mammalian cells by LMB is ascribable to inhibition of the mammalian CRM1 function.

\section{CRM1 is essential for nuclear export of proteins}

We showed that LMB inhibited nuclear export of not only HIV-1 Rev but also other cellular proteins such as MAPKK and PKIa, when GFP-fusion proteins bearing NESs were injected into the nucleus ${ }^{16,17}$. . These results indicate that LMB is a general nuclear export inhibitor. To confirm that CRM1 is the target of LMB, we tested whether LMB binds directly to $h C R M 1^{17)}$. A biotinylated derivative of LMB binds to a protein that is reactive with an antihCRM1 antibody but not in the presence of 100 fold excess of LMB. Microinjection of a purified anti-hCRMl antibody as well as LMB specifically inhibited nuclear export of NEScontaining proteins. Furthermore, the fission yeast $\mathrm{crml}$ mutant was defective in the nuclear export of NES-fused proteins but not in the 
import of NLS-fused proteins. Interestingly, the protein containing both the NES and the NLS that would shuttle between the nucleus and the cytoplasm was highly accumulated in the nucleus in the $\mathrm{cm} / \mathrm{l}$ mutant cells or the cells treated with LMB. Finally, we showed interaction of CRM1 with the NES by employing the immobi- lized NES and HeLa cell extracts. This association was disrupted by adding LMB or the purified anti-hCRMl antibody ${ }^{17}$. These results together with those by others show that CRM1, like importin $\beta$ for import, is an essential factor for nuclear export of proteins in all eukaryotes ${ }^{16-}$ 20). Thus, CRM1 has been called exportin 1 .

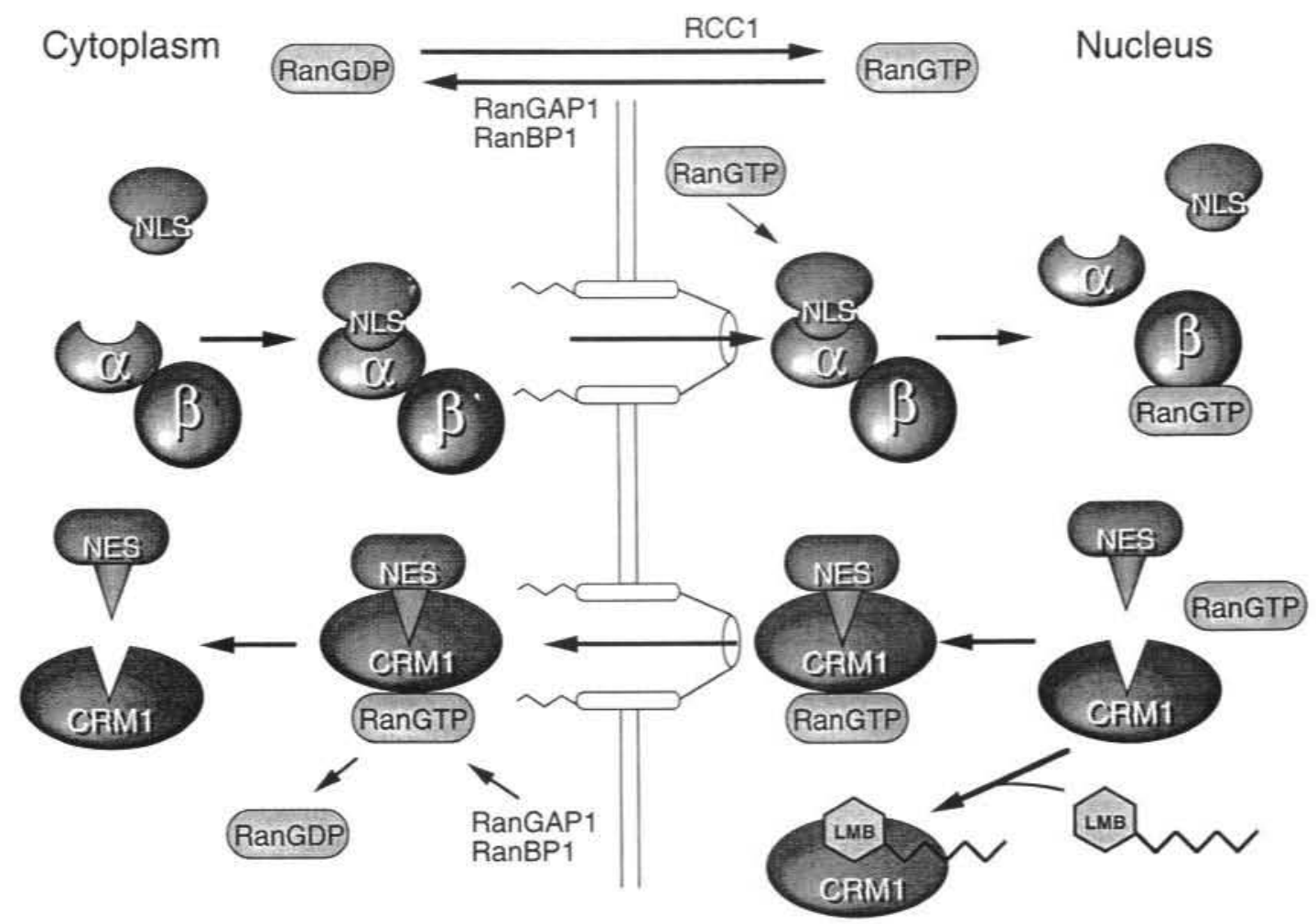

Fig. 2. A model for nucleo-cytoplasmic transport of proteins and molecular action of LMB.

5. Present model for nuclear protein import and export

A model for nuclear protein import and export was illustrated in Fig. 2. The import of proteins into the nucleus occurs through the nuclear pore complexes (NPC) which allow diffusion of small molecules and can accommo- date the active transport of particles as large as several million dalton in weight ${ }^{21,22)}$. The active import is energy-dependent and is conferred by virtue of the NLS that is rich in basic amino acids. The translocation of the NLS-bearing protein into the nucleoplasm is mediated by the saturable import machinery that 
recognizes basic $\mathrm{NLSs}^{23}$. The import receptor for the NLS was identified to be a heterodimeric complex of importin $\alpha$ and importin $\beta$ (also known as karyopherin $\alpha$ and $\beta$ or PTACs). Importin $\alpha$ recognizes and binds basic NLSs, while importin $\beta$ mediates docking of the cargoimportin complex with the NPC, in a temperature-independent manner. Translocation into the nucleoplasm is mediated by the Ran GTPase cycle, and requires energy in the form of GTP hydrolysis ${ }^{24,25)}$. Importin $\beta$ binds directly to the NPC and Ran-GTP. In turn, Ran-GTP binding to importin $\beta$ dissociates the importin $\alpha$ $\beta$ heterodimer ${ }^{26,27)}$. Ran-GTP binds to the amino terminus of importin $\beta$, whereas importin $\alpha$ binds to the carboxy-terminal region of importin $\beta$. Release of importin $\alpha$ upon Ran-GTP binding to importin $\beta$ is therefore probably explained by a conformational change in importin $\beta$.

Ran is a Ras homolog in the nucleus, which switches between a GDP-bound and a GTPbound state by nucleotide exchange and GTP hydrolysis, like all G proteins. RCC1 (regulator of chromosome condensation 1) is a major guanine nucleotide exchange factor (GEF) for Ran and generates Ran-GTP ${ }^{28}$. RanGAP1 is the GTPase-activating protein and causes conversion of Ran-GTP into Ran-GDP ${ }^{29}$. A characteristic feature of the Ran system is the asymmetric distribution of Ran-GTP and RanGDP. RCC1 is chromatin-bound and generates Ran-GTP in the nucleus ${ }^{30)}$. In contrast, RanGAP1 is cytoplasmic, and therefore depletes Ran-GTP from the cytoplasm ${ }^{31}$. This asymmetric distribution of Ran explains why the RanGTP-mediated dissociation of importin heterodimer occurs specifically in the nucleus that follows cargo translocation into the nucleus ${ }^{32}$.

As described above, CRM1 was identified to be the NES receptor and renamed as exportin 1 . CRM1/exportin 1 shares low but significant homology to importin $\beta^{33},{ }^{34)}$. CRMl/exportin 1 is shown to convey NES-bearing proteins through the NPC in a Ran-GTP-dependent manner. It is likely that GTP hydrolysis that may occur in the cytoplasm dissociates the cargoCRM1/exportin 1 complex. In contrast to the import system mediated by the importin heterodimer, CRM1/exportin 1 alone can associate both with a cargo and Ran-GTP (Fig. 2). LMB binds to CRM1/exportin 1, thereby inhibiting cargo loading and subsequent nuclear export.

\section{Role for LMB as a chemical probe}

Increased evidence has suggested that many signaling molecules have the NLS or the NES or both, by which they relocalize in response to various stimuli. For instance, unstimulated MEK binds and anchors MAPK in the cytoplasm by its NES. The phosphorylated, activated MAPK can be released from MEK and enter the nucleus, although it is still unclear how MAPK dissociates from the complex ${ }^{16}, 35$ ). NES in cyclin $B 1^{36,37)}$ is inactivated by phosphorylation of the amino-terminal region of the protein, which allows nuclear translocation of cyclin $\mathrm{B} 1 / \mathrm{Cdc} 2$ complex upon the onset of mitosis ${ }^{38}$. On the other hand, MAPKAP kinase 2, an NLSbearing nuclear substrate of MEK and p38, is exported from the nucleus by phosphorylation of a threonine residue (T317) that is overlapped with the potential NES ${ }^{39}$. Recently, we showed that Papl, an S. pombe bZip transcription factor, contained both an NLS and an NES, of which transcriptional activity was negatively regulated by CRM1/exportin 1dependent cytoplasmic localization. Surprisingly, the nuclear export of Papl was abolished by a subset of oxidants, suggesting that the Pap 1 NES is sensitive to oxidative stress (unpublished results). LMB played particularly important roles in identification of the NES in these studies.

Although the sequence requirements for NES function are only loosely defined, the consensus sequence for the functional Rev/Rex NES has been proposed to be $\mathrm{L}-\mathrm{X}_{2 \cdot 3}-\mathrm{L} / \mathrm{/} / \mathrm{F} / \mathrm{V} / \mathrm{M}-\mathrm{X}_{2 \cdot 3}-\mathrm{L}-\mathrm{X}-$ $\mathrm{L}^{40)}$. Database searches with this motif identify a large number of proteins. For instance, 
Cyclin A

Cyclin B1

Cyclin D3

Cyclin E

MAPKK

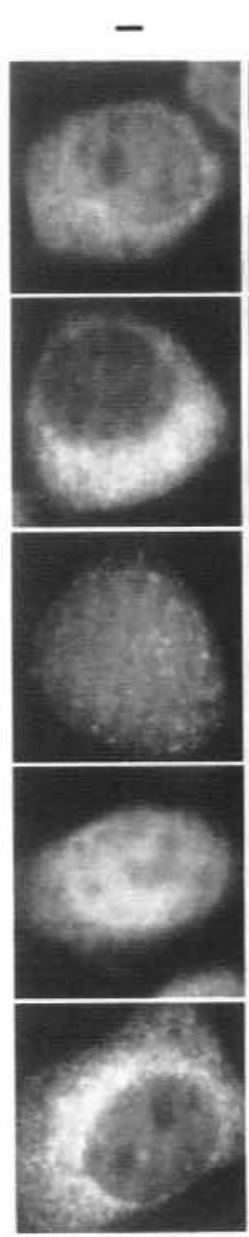

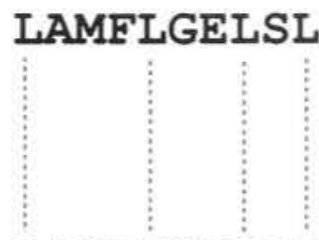

LCQAFSDVIL

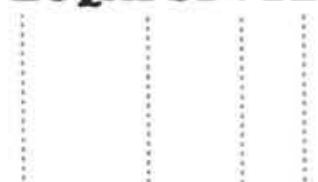

LAFILHRLSL

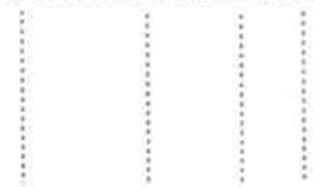

LKWRLSPLTI

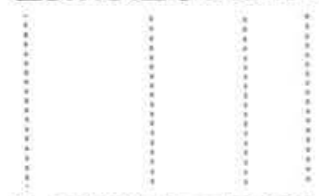

LQKKLEELEL
+LMB

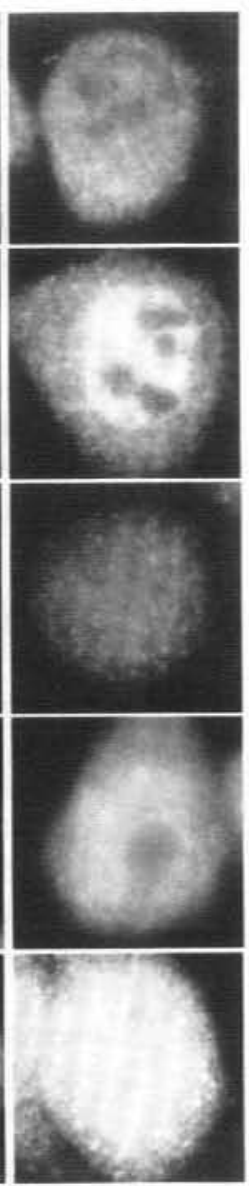

Fig. 3. The use of LMB for identification of NESs in the regulatory proteins. Cyclins A, B1, D3, and $\mathrm{E}$ contain sequences highly similar to the NES consensus. However, immunofluorescent staining showed that nuclear accumulation of cyclin B1 was observed, while subcellular localization of other cyclins was unchanged, indicating that only the sequence in cyclin B1 is functional as an NES. Changes in the localization of MAPKK that contains an NES was also shown as a positive control.

the sequences matched with the motif are seen in cyclins A, B1, D3 and E. However, only the sequence in cyclin B1 was functional, when the nuclear export activity was assessed by LMB (Fig. 3). It is likely that other constrains on this type of NES have yet to be defined. Thus, LMB will serve broadly as an important tool for identifying the proteins of being exported in both lower and higher eukaryotes.

The mechanisms by which LMB induces G1 and/or G2 arrest of the cell cycle and antitumor effects are still unclear. CAN/Nup214, the product of a human oncogene associated with myeloid leukemia that is activated by trans- 
location $(6 ; 9)$ with other genes ${ }^{41}$, was shown to be complexed with CRM1/exportin $1^{42)}$. CAN/ Nup214 is a member of nucleoporins, the components of the NPC ${ }^{43)}$. This finding suggests that nucleo-cytoplasmic transport itself is one of the targets of oncogenesis. It is likely that the cell cycle arrest and tumor suppression by LMB and its derivatives come from the inhibition of the nuclear export of some regulatory proteins involved cell cycle control and oncogenesis. Identification of the substrate proteins responsible for the cell cycle arrest and antitumor activity is obviously important for future development of antitumor agents.

\section{ACKNOWLEDGMENTS}

We thank Dr. K. Nishi, Pharmaceutical Discovery Research Division, Takeda Chemical Industries, Ltd., Dr. Y. Yoneda, Department of Anatomy and Cell Biology, Osaka University Medical School, Suita, Osaka 565, M. Yanagida, Department of Biophysics, Graduate School of Science, Kyoto University, Sakyo-ku, Kyoto 606-01, Japan, Dr. B. Wolff, Novartis Forschungsinstitut, Brunner strasse 59, A-1235 Vienna, Austria, and Dr. S. Khochbin, INSERM U309, Institut Albert Bonniot, Faculte de Medecine, La Tronche, France, for collaboration. We are grateful to Dr. T. Beppu, Department of Applied Biological Sciences, Nihon University, for discussion and encouragement.

\section{REFERENCES}

1) Hamamoto, T., S. Gunji, H. Tsuji \& T. Beppu.: Leptomycins $\mathrm{A}$ and $\mathrm{B}$, new antifungal antibiotics. 1. Taxonomy of the producing strain and their fermentation, purification and characterization. J. Antibiotics 36: 639645, 1983.

2) Hamamoto, T., H. Seto \& T. Beppu.: Leptomycins $\mathrm{A}$ and $\mathrm{B}$, new antifungal antibiotics. II. Structure elucidation. J. Antibiotics 36: 646-650, 1983.

3) Hamamoto, T., T. Uozumi \& T. Beppu.:
Leptomycins A and B, new antifungal antibiotics. III. Mode of action of leptomycin $\mathrm{B}$ on Schizosaccharomyces pombe. J. Antibiotics 38: 1573-1580, 1985.

4) Yoshida, M., M. Nishikawa, K. Nishi, K. Abe, S. Horinouchi \& T. Beppu.: Effects of leptomycin B on the cell cycle of fibroblasts and fission yeast cells. Exp. Cell Res. 187: 150-156, 1990.

5) Komiyama, K., K. Okada, S. Tomisaka, I. Umezawa, T. Hamamoto \& T. Beppu.: Antitumor activity of leptomycin B. J. Antibiotics 38: 427-429, 1985.

6) Komiyama, K., K. Okada, Y. Hirokawa, K. Masuda, S. Tomisaka \& I. Umezawa.: Antitumor activity of a new antibiotic, kazusamycin. J. Antibiotics 38: 224-229, 1985.

7) Hayakawa, Y., K. Adachi \& N. Komeshima.: New antitumor antibiotics, anguinomycins A and B. J.Antibiotics 40: 1349-1352, 1987.

8) Nishi, K., M. Yoshida, M. Nishimura, M. Nishikawa, M. Nishiyama, S. Horinouchi \& T. Beppu.: A leptomycin B resistance gene of Schizosaccharomyces pombe encodes a protein similar to the mammalian Pglycoproteins. Mol. Micorbiol. 6: 761$769,1992$.

9) Adachi, Y. \& M. Yanagida.: Higher order chromosome structure is affected by coldsensitive mutations in a Schizosaccharomyces pombe gene $\mathrm{crml}$ which encodes a $115-\mathrm{kD}$ protein preferentially localized in the nucleus and at its periphery. J. Cell Biol. 108: 1195-1207, 1989.

10) Toda, T., M. Shimanuki, Y. Saka, H. Yamano, Y. Adachi, M. Shirakawa, Y. Kyogoku \& M. Yanagida: Fission yeast pap1-dependent transcription is negatively regulated by an essential nuclear protein, crm 1. Mol. Cell. Biol. 12: 5474-5484, 1992.

11) Kumada, K., M. Yanagida \& T. Toda.: Caffeine-resistance in fission yeast is caused by mutations in a single essential gene, $\mathrm{crml}$ : Mol. Gen. Genet. 250: 59-68, 1996.

12) Nishi, K., M. Yoshida, D. Fujiwara, M. Nishikawa, S. Horinouchi \& T. Beppu.: Leptomycin B targets a regulatory cascade of $\mathrm{crml}$, a fission yeast nuclear protein, involved in control of higher order chromosome structure and gene expression. J. Biol. Chem. 269: 6320-6324, 1994.

13) Kudo, N., S. Khochbin, K. Nishi, K. Kitano, M. Yanagida, M. Yoshida \& S. Horinouchi.: 
Molecular cloning and cell cycle-dependent expression of mammalian CRM1, a protein involved in nuclear export of proteins. J. Biol. Chem. 272: 29742-29751, 1997.

14) Wolff, B., J.-J. Sanglier \& Y. Wang.: Leptomycin B is an inhibitor of nuclear export: inhibition of nucleo-cytoplasmic translocation of the human immunodeficiency virus type 1 (HIV-1) Rev protein and Rev-dependent mRNA. Chem. Biol. 4: 139-147, 1997.

15) Fischer, U., J. Huber, W. C. Boelens, I. W. Mattaj \& R. Lührmann.: The HIV-1 Rev activation domain is a nuclear export signal that accesses an export pathway used by specific cellular RNAs. Cell 82: 475-483, 1995.

16) Fukuda, M., S. Asano, T. Nakamura, M. Adachi, M. Yoshida, M. Yanagida \& E. Nishida.: CRM1 is responsible for intracellular transport mediated by the nuclear export signal. Nature 390: 308-311, 1997.

17) Kudo, N., B. Wolff, T. Sekimoto, E. P. Schreiner, Y. Yoneda, M. Yanagida, S. Horinouchi \& M. Yoshida.: Leptomycin B inhibition of signal-mediated nuclear export by direct binding to CRM1. Exp. Cell Res, 242: 540-547, 1998.

18) Fornerod, M., M. Ohno, M. Yoshida \& I. W. Mattaj.: CRM1 is an export receptor for leucine-rich nuclear export signals. Cell 90: 1051-1060, 1997.

19) Ossareh-Nazari, B., F. Bachelerie \& C. Dargemont.: Evidence for a role of CRM1 in signal-mediated nuclear protein export. Science 278: 141-144, 1997.

20) Stade, K., C. S. Ford, C. Guthrie \& K. Weis. Exportin $1(\mathrm{Crm} 1 \mathrm{p})$ is an essential nuclear export factor. Cell 90: 1041-1050, 1997.

21) Dworetzky, S. I., R. E. Lanford \& C. M. Feldherr.: The effects of variations in the number and sequence of targeting signals on nuclear uptake. J. Cell Biol. 107: 1279 . 1287, 1988.

22) Rout, M. P. \& S. R. Wente.: Pores for thought: nuclear pore complex proteins. Trends Cell Biol. 4: 357-365, 1994.

23) Dingwall, C. \& R. A. Laskey.: Nuclear targeting sequences - a consensus? Trends Biochem. Sci. 16: 478-481, 1991.

24) Görlich, D. \& I. W. Mattaj.: Nucleocytoplasmic transport. Science 271: 15131518, 1996.

25) Melchior, R. \& L. Gerace.: Mechanisms of nuclear protein import. Curr. Opin. Cell Biol. 7: 310-318, 1995.

26) Görlich, D., N. Pante, U. Kutay, U. Aebi \& F. R. Bischoff.: Identification of different roles for RanGDP and RanGTP in nuclear protein import. EMBO J. 15: 5584-5594, 1996.

27) Rexach, M. \& G. Blobel.: Protein import into nuclei: association and dissociation reactions involving transport substrate, transport factors, and nucleoporins. Cell 83: 683-92, 1995.

28) Bischoff, F. R. \& H. Ponstingl.: Catalysis of guanine nucleotide exchange on Ran by the mitotic regulator $\mathrm{RCCl}$. Nature 354: 8082, 1991.

29) Bischoff, F. R., H. Krebber, T. Kemph, I. Hermes \& H. Ponstingl.: Human RanGTPase activating protein RanGAP1 is a homolog of yeast RNA1p involved in messenger RNA processing and transport. Proc. Natl. Acad. Sci. USA 92: 1749-1753, 1995.

30) Ohtsubo, M., H. Okazaki \& T. Nishimoto.: The $\mathrm{RCC} 1$ protein, a regulator for the onset of chromosome condensation locates in the nucleus and binds to DNA. J. Cell Biol. 109: 1389-1397, 1989.

31) Hopper, A. K., H. M. Traglia \& R. W. Dunst.: The yeast RNA1 gene product necessary for RNA processing is located in the cytosol and apparently excluded from the nucleus. J. Cell Biol. 111: 309-321, 1990.

32) Izaurralde, E., U. Kutay, C. von Kobbe, I. W. Mattaj \& D. Gorlick.: The asymmetric distribution olf the constituents of the Ran system is essential for transport into and out of the nucleus. EMBO J. 16: 6535-6547, 1997.

33) Görlich, D., F. Vogel, A. D. Mills, E. Hartmann \& R. A. Laskey.: Distinct functions for the two importin subunits in nuclear protein import. Nature 377: 246-248, 1995.

34) Moroianu, J., M. Hijikata, G. Blobel \& A. Radu.: Mammalian karyopherin $\alpha 1 \mathrm{~b}$ and $\alpha_{2} \mathrm{~b}$ heterodimers: $\alpha 1$ or $\alpha 2$ subunit binds nuclear localization sequence and $\beta$ subunit interacts with peptide repeat containing nucleoporins. Proc. Natl. Acad. Sci. USA 92: 6532-6536, 1995.

35) Fukuda, M., I. Gotoh, Y. Gotoh \& E. Nishida.: Cytoplasmic localization of mitogen-activated protein kinase kinase directed by its $\mathrm{NH}_{2}^{-}$ terminal, leucine-rich short amino acid sequence, which acts as a nuclear export signal. J. Biol. Chem. 271: 20024-20028, 1996. 
36) Hagting, A., C. Karlsson, P. Clute, M. Jackman \& J. Pines.: MPF localization is controlled by nuclear export. EMBO J. 17: 4127-4138, 1998.

37) Toyoshima, F., T. Moriguchi, A. Wada, M. Fukuda \& E. Nishida.: Nuclear export of cyclin B1 and its possible role in the DNA damage- induced $\mathrm{G} 2$ checkpoint. EMBO J. 17: 2728-2735, 1998.

38) Yang, J., E. S. Bardes, J. D. Moore, J. Brennan, M. A. Powers \& S. Kornbluth.: Control of cyclin B1 localization through regulated binding of the nuclear export factor CRM1. Genes Dev. 12: 2131-2143, 1998.

39) Engel, K., A. Kotlyarov \& M. Gaestel.: Leptomycin B-sensitive nuclear export of MAPKAP kinase 2. EMBO J. 17: 33633371, 1998.

40) Bogerd, H. P., R. A. Fridell, R. E. Benson, J. Hua \& B. R. Cullen.: Protein sequence requirements for function of the human T-cell leukemia virus type 1 Rex nuclear export signal delineated by a novel in vivo random- ization-selection assay. Mol. Cell. Biol. 16: 4207-4214, 1996.

41) von Lindern, M., S. van Baal, J. Wiegant, A. Raap, A. Hagemeijer \& G. Grosveld.: Can, a putative oncogene associated with myeloid leukemogenesis, may be activated by fusion of its $3^{\prime}$ half to different genes: characterization of the set gene. Mol. Cell. Biol. 12: 3346$3355,1992$.

42) Fornerod, M., J. v. Deursen, S. van. Baal, A. Reynolds, D. Davis, K. G. Murti, J. Fransen \& G. Grosveld.: The human homologue of yeast CRM1 is in a dynamic subcomplex with CANNup214 and a novel nuclear pore component Nup88. EMBO J. 16:807-816, 1997.

43) Fornerod, M., J. Boer, S. van Baal, H. Morreau \& G. Grosveld.: Interaction of cellular proteins with the leukemia specific fusion proteins DEK-CAN and SET-CAN and their normal counterpart, the nucleoporin CAN. Oncogene 13: 1801-1808, 1996. 